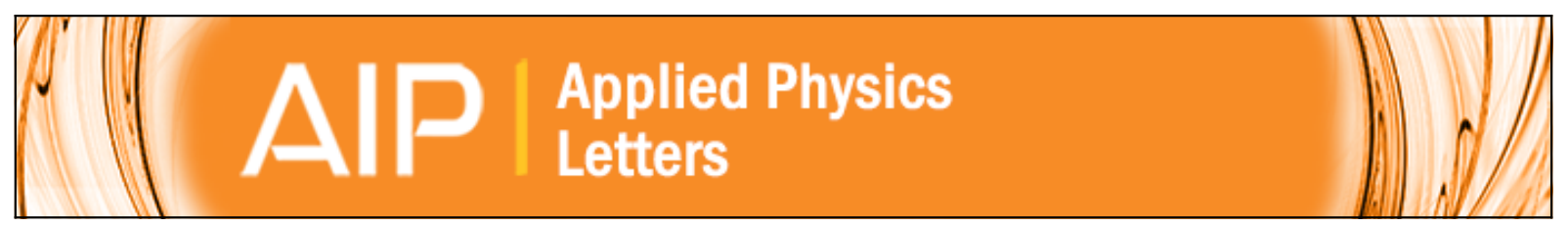

\title{
All-fiber passively mode-locked thulium-doped fiber ring laser using optically deposited graphene saturable absorbers
}

QingQing Wang, Tong Chen, Botao Zhang, Mingshan Li, Yongfeng Lu, and Kevin P. Chen

Citation: Applied Physics Letters 102, 131117 (2013); doi: 10.1063/1.4800036

View online: http://dx.doi.org/10.1063/1.4800036

View Table of Contents: http://scitation.aip.org/content/aip/journal/apl/102/13?ver=pdfcov

Published by the AIP Publishing

\section{Articles you may be interested in}

Tunable graphene saturable absorber with cross absorption modulation for mode-locking in fiber laser Appl. Phys. Lett. 105, 041901 (2014); 10.1063/1.4891645

Mechanical exfoliation of graphene for the passive mode-locking of fiber lasers

Appl. Phys. Lett. 99, 121107 (2011); 10.1063/1.3641419

Graphene mode locked, wavelength-tunable, dissipative soliton fiber laser

Appl. Phys. Lett. 96, 111112 (2010); 10.1063/1.3367743

Large energy soliton erbium-doped fiber laser with a graphene-polymer composite mode locker

Appl. Phys. Lett. 95, 141103 (2009); 10.1063/1.3244206

Chaotic dynamics of a passively mode-locked soliton fiber ring laser

Chaos 16, 013128 (2006); 10.1063/1.2173049

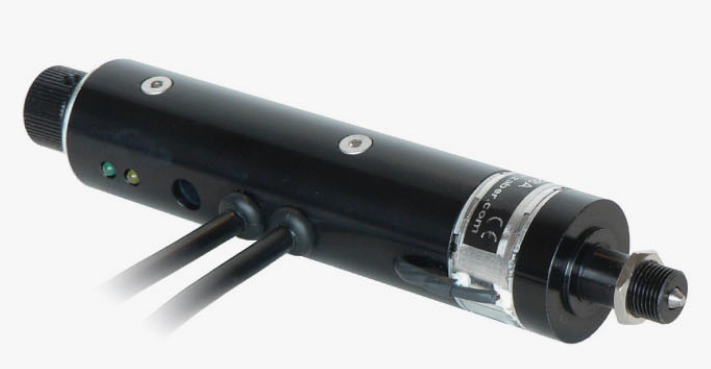

Automate your set-up with 


\title{
All-fiber passively mode-locked thulium-doped fiber ring laser using optically deposited graphene saturable absorbers
}

\author{
QingQing Wang, ${ }^{1}$ Tong Chen, ${ }^{1}$ Botao Zhang, ${ }^{1}$ Mingshan Li, ${ }^{1}$ Yongfeng Lu, ${ }^{2}$ \\ and Kevin P. Chen ${ }^{1, a)}$ \\ ${ }^{1}$ Department of Electrical Engineering, University of Pittsburgh, Pittsburgh, Pennsylvania 15261, USA \\ ${ }^{2}$ Department of Electrical Engineering, University of Nebraska-Lincoln, Lincoln, Nebraska 68588-0511, USA
}

(Received 14 September 2012; accepted 21 March 2013; published online 5 April 2013)

\begin{abstract}
An all-fiber passively mode-locked thulium-doped fiber ring oscillator is constructed using optically deposited few layer graphene micro-sheets as the saturable absorber (SA). The mode-lock operation was achieved by $130-\mathrm{mW}$ pump power at $1.5-\mu \mathrm{m}$. The fiber oscillator produces $2.1-\mathrm{ps}$ soliton pulse output with $80-\mathrm{pJ}$ per pulse energy. The 3-dB bandwidth of the laser output was measured as 2.2-nm. The RF signal-to-noise ratio of $50-\mathrm{dB}$ and sub $20-\mathrm{Hz} 3-\mathrm{dB}$ bandwidth of the laser output confirms the stable laser operation with low time jittering. This paper shows that graphene can be an effective saturable absorber for the development of mid-IR fiber mode-locked laser. (C) 2013 American Institute of Physics. [http://dx.doi.org/10.1063/1.4800036]
\end{abstract}

In recent years, a great deal of research efforts have been devoted into the development of ultra-short pulsed light sources based on various rare-earth doped fibers. Compared with bulk solid state lasers, fiber lasers possess many desirable characteristics including compactness, low cost, ease to build, and good reliability under harsh environments. While ultrafast fiber lasers using ytterbium and erbium doped fibers have flourished, ${ }^{1,2}$ many researchers now place their research focuses on the development of fiber lasers at $2-\mu \mathrm{m}$ using thulium-doped fibers (TDFs) and longer wavelength for a wide range of applications in remote sensing, medical diagnostics, military, etc.

The first passively mode-locked TDF laser was reported by Nelson was realized using nonlinear polarization evolution (NPE), which was operated in solitary mode. ${ }^{3}$ Noise-like pulses were also reported based on the same mechanism. ${ }^{4}$ Alternatively, InGaAs quantum and single-wall carbon nanotube (SWCNT) saturable absorbers (SAs) have also been used to construct mode-locked fiber lasers. ${ }^{5-7}$

Graphene, a monolayer of carbon atoms arranged in a two-dimensional honeycomb pattern, has been extensively studied recently and exhibits saturable absorption effects under strong optical excitation. ${ }^{8}$ Compared with other SAs, graphene-based saturable absorbers (GSAs) own many merits including low fabrication cost, moderate saturable modulation depth, moderate damage intensity threshold, and ultrafast recovery time. ${ }^{9,10}$ Since 2009 , a number of papers have been reported to harness this unique nonlinear optical property of graphene in construction of mode-locked fiber lasers. So far, GSAs have been reported and utilized to construct passively mode-locked ytterbium and erbium doped fiber lasers, ${ }^{10-12}$ and very recently passively Q-switched and mode-locked $\mathrm{Tm}$-doped lasers at $2-\mu \mathrm{m}$ by graphene based saturable absorbers have also been demonstrated. ${ }^{13,14}$

One of interesting characteristics of graphene saturable absorber (GSAs), in comparison to semiconductor-based devices, is the ultra-broad operational bandwidth. This unique trait of graphene enables the development of ultrafast

a)email: pec9@pitt.edu photonic applications in mid-IR spectral ranges, while midIR ultrafast components are not easily attainable.

In this Letter, we explore this opportunity by demonstrating a $2-\mu \mathrm{m}$ all-fiber GSA passively mode-locked TDF ring oscillator. To our best knowledge, this is first modelocked TDF fiber ring oscillator realized by optically deposited GSA, which would be valuable to extend graphene materials' applications.

The graphene film for the TDF laser in this work was prepared by an ultrasonic exfoliation method. The graphite flakes (Sigma-Aldrich) were first hand mortared for 15-min. $3 \%$ wt. mortared graphite flakes were dispersed into $\mathrm{N}$-dimethylformaminde (DMF) solvent and ultrasonically bathed for $48 \mathrm{~h}$ at room temperature. The dispersion solution was then centrifuged for $30 \mathrm{~min}$ at $1000 \mathrm{rpm}$ and only the top portion of transparent liquid containing graphene microsheets with lightly grey color was harnessed for further process.

Figure 1 shows the Raman spectra for the starting graphite flake (top) and the derived dispersion solution after the process (bottom). The Raman spectra were acquired using 633-nm laser excitation. The higher $\mathrm{D} / \mathrm{G}$ band intensity ratio

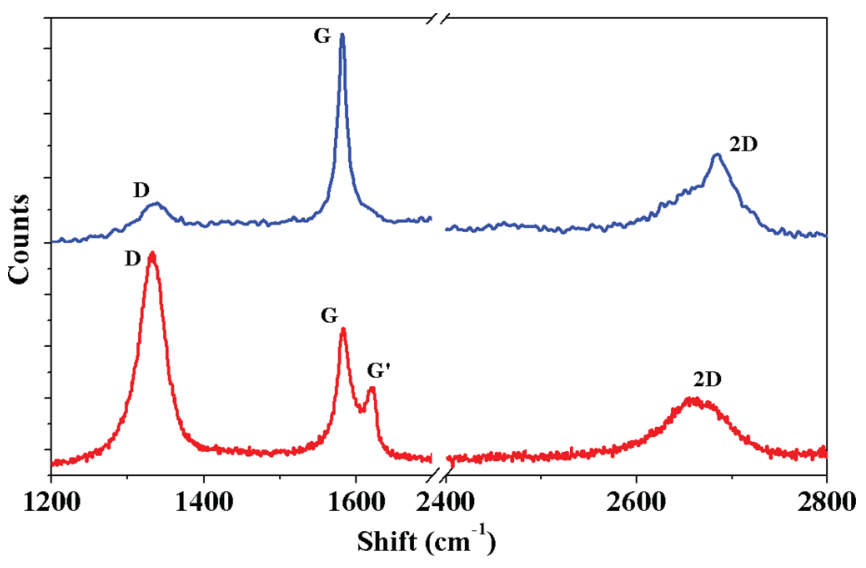

FIG. 1. Raman spectra for the starting graphite flakes (top) and the derived graphene dispersion (bottom). 
in the dispersion solution suggests the breakdown of the graphite flake after the process. The relative shift of the 2D peak and the ratio of $2 \mathrm{D} / \mathrm{G}$ peaks indicate the formation of single-layer and few-layer graphene sheets in the dispersion solution. It was reported that this process can produce the dispersion solution containing $10 \%$ mono-layer graphene sheets and up to $80 \%$ of few-layer graphene sheet containing less than five graphene layers. ${ }^{15}$ Both of mono-layer and few-layer graphene can be used as optical saturable absorbers, ${ }^{9}$ and the mean number of layers of the developed graphene micro-sheets is estimated as 4 based on the Raman spectrum.

The dispersed graphene micro-sheets in the liquid solution were then optically deposited onto the end tip of single mode fiber. The detail of this optical deposition approach can be found in Ref. 16. In our optical deposition setup, a 200-mW 1550-nm light source from an erbium-doped fiber amplifier (EDFA) was used to optically trap few-layer graphene onto the fiber tip. An enhanced back-reflection can be registered by an optical spectrum analyzer (OSA) when the graphene film was successfully deposited. Once the quality of the film was confirmed, the graphene-coated fiber was then connected with a single-mode fiber patch cord using a bare fiber connector and a mating sleeve to accomplish a desired GSA module. The typical linear absorbance of the fabricated GSA is measured at the order of $20 \%$ at $2-\mu \mathrm{m}$, and the insertion loss of the mating is around $0.7-\mathrm{dB}$. The typical transmittance curve at $1945-\mathrm{nm}$ as the function of average incident power is shown in Figure 2(a), for which an amplified mode-locked laser operated at central wavelength of 1945-nm with $12.8-\mathrm{MHz}$ repetition rate, $\sim 2$-ps temporal duration was used as the probe. The transmittance is consistently around $62 \%$ when the average pump power is below 3 - $\mathrm{mW}$ and increases by $\sim 4 \%$ as the pump power rises to 6 $\mathrm{mW}$ due to the saturable absorption for the few-layer graphene deposited on the fiber tip. Previous works report that mono-layer graphene could exhibit larger modulation depth at $1550-\mathrm{nm} .{ }^{9}$

The fabricated GSA was then inserted into an all-fiber TDF ring cavity as depicted in Figure 2(b). The active fiber in the laser cavity is a piece of $35-\mathrm{cm}$ long single mode Tmdoped silica fiber with $5-\mu \mathrm{m}$ core diameter and a numerical
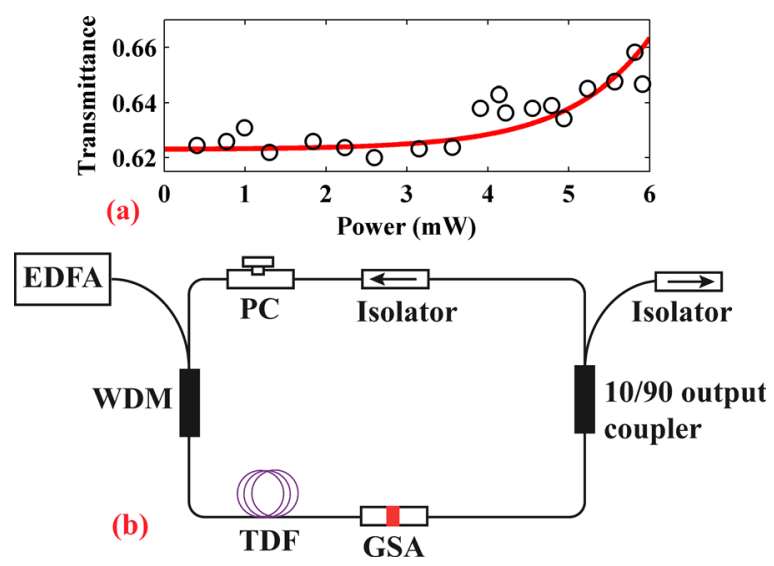

FIG. 2. (a) Typical transmittance curve of the fabricated GSA as function of pump power and (b) scheme of the TDF ring cavity, based on fabricated GSA. aperture of 0.23 . A $1550-\mathrm{nm} / 1900-\mathrm{nm}$ wavelength division multiplexing (WDM) couplers is used to couple pump light into the cavity. An amplified spontaneous emission light source from an EDFA is fusion spliced to the WDM to provide forward pumping. The laser output from the oscillator is coupled out through the $10 \%$ port of a $10 / 90$ output coupler. A polarization insensitive isolator with $10 \%$ insertion loss and $>35-\mathrm{dB}$ isolation at $2-\mu \mathrm{m}$ wavelength is fusion spliced into the cavity to enforce a counterclockwise unidirectional ring. A second isolator at $2-\mu \mathrm{m}$ wavelength is also fusion spliced to the output port to suppress back reflection into the cavity. An in-line polarization controller (PC) is inserted into the cavity to examine the polarization effects on the modelocking operation. It was intended to balance the intrinsic weak birefringence of single-mode fibers used to construct the laser cavity. However, our experimental results showed that the mode-locking operation is virtually independent of the tuned position of the PC. The laser cavity shown in Fig. 2(b) consists of $11.5-\mathrm{m}$ of SMF-28e fiber. The anomalous dispersion of the SMF-28e fiber at 1.9- $\mu \mathrm{m}$ is measured to be $-67-\mathrm{ps}^{2} / \mathrm{km}$ using a white-light interferometry technique. ${ }^{17}$ The dispersion of the TDF used in the experiment is estimated to be $-12-\mathrm{ps}^{2} / \mathrm{km}$. The net dispersion in the cavity is calculated as $-0.77-\mathrm{ps}^{2}$. The small amount of dispersion due to the optical isolator is neglected.

Stable CW mode-locking of the oscillator is self-started as the pump ramps up to $130-\mathrm{mW}$. This threshold is lower than those values previously reported for SWCNT modelocked fiber lasers operated under the same soliton regime (280-mW for Ref. 6 and 320-mW for Ref. 7). The fiber cavity dispersion and fiber length play could contribute to the lower threshold. However, previous experiments also reported that mode-lock threshold for graphene saturable absorber is lower than those using SWCNT at 1550-nm using the identical fiber laser configuration. ${ }^{16}$ In Figure 3, the typical optical spectrum of the mode-locked output under the $130-\mathrm{mW}$ pumping power is demonstrated with a resolution of $0.1-\mathrm{nm}$ on a logarithm scale. The central wavelength is 1953.3-nm, and the FWHM is 2.2-nm. Strong Kelly sidelobes due to the inference of dispersion waves are clearly indicative of the solitary operation of the laser. ${ }^{18}$ The modelocking operation is not affected by tuning the position of the PC, which confirms that the nonlinear amplitude modulation

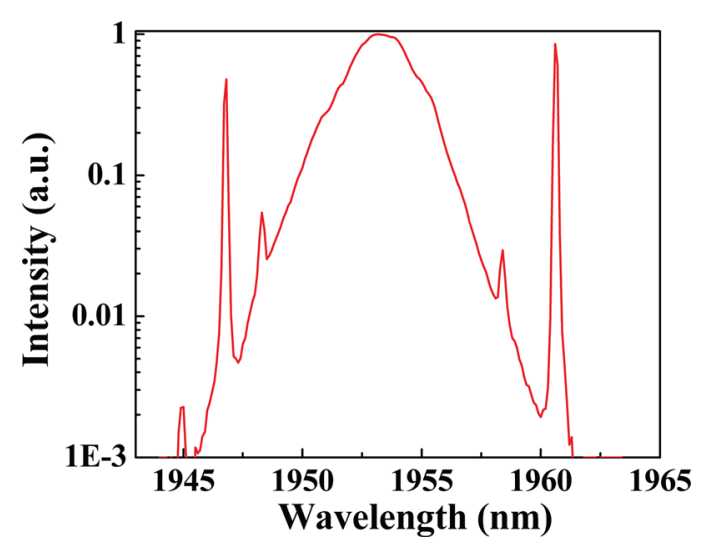

FIG. 3. Typical spectrum of the mode-locked laser on logarithm scale. 


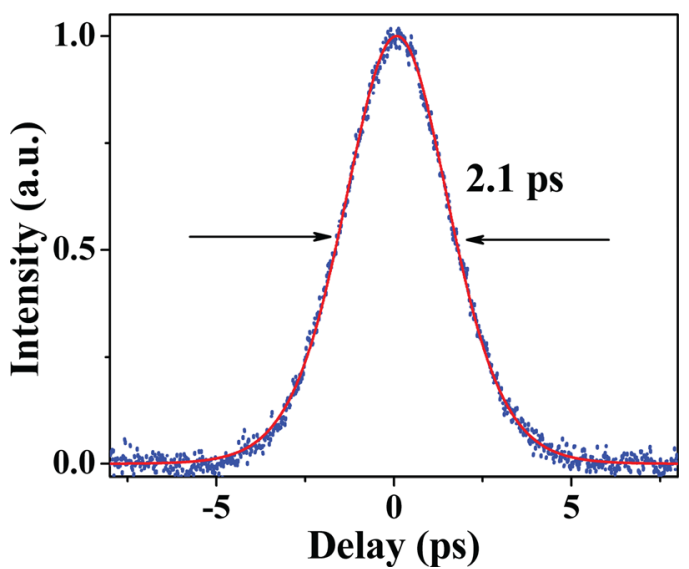

FIG. 4. Intensity autocorrelation trace of GSA mode-locked TDF laser.

in the ring cavity is due to the fabricated GSA's saturable absorption other than any unbalanced NPE effect.

The pulse width is determined by a home-built intensity autocorrelator as shown in Figure 4. The autocorrelation measurement yields 2.1-ps pulse width (FWHM) assuming a hyperbolic-secant soliton pulse shape. Considering the 2.2$\mathrm{nm} 3-\mathrm{dB}$ bandwidth, the time bandwidth product (TBP) is calculated as 0.363 , which is slightly larger than the transform-limit value 0.315 .

A 7-GHz fast InGaAs detector (EOTECH ET-5010F) is used to measure the radiofrequency (RF, TEKTRONIX 3408A) characteristics of the mode-locked operation. High resolution RF spectrum of the GSA mode-locked pulse trains at the fundamental repetition rate is shown in Figure 5. The center frequency is $16.937-\mathrm{MHz}$, which is consistent of the fundamental repetition rate of the cavity. This confirms that one pulse is produced per round trip. The signal to noise ratio (SNR) was measured as $50 \mathrm{~dB}$, and the $3-\mathrm{dB}$ bandwidth of the RF spectrum was sub the RF resolution of the instrument at 20-Hz. These results are comparable with those reported for GSA mode-locked erbium fiber laser using similar fabrication process. ${ }^{16}$ We notice that SNR for Tm-doped or Er-doped mode-locked fiber lasers can reach $80-\mathrm{dB}$ if SESAMS were used. Therefore, the low SNR could come from the quality of graphene as mode-locking elements. Wide range RF spectrum from 0 to $2-\mathrm{GHz}$ is shown as the

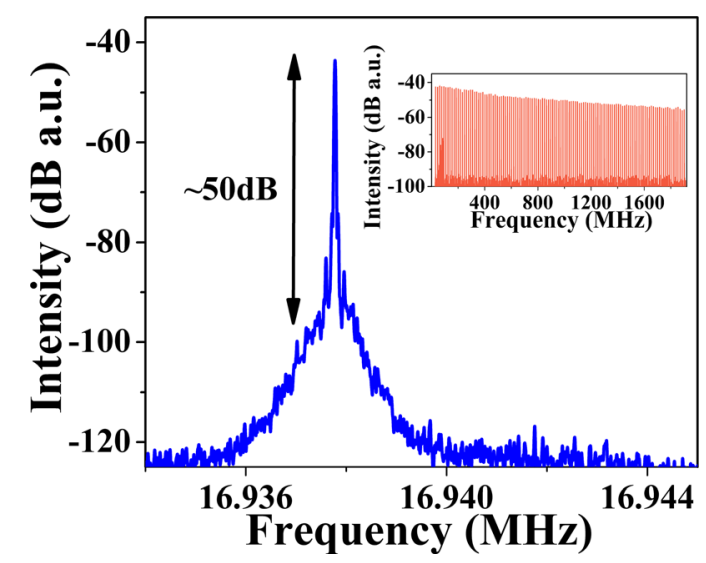

FIG. 5. RF spectrum around the fundamental repletion rate; inset: RF spectrum from 0 to $2-\mathrm{GHz}$. inset of Figure 5. These measurement results indicate low time jittering of the pulse trains. The average output power from the $10 \%$ output port is measured as $1.41-\mathrm{mW}$. Given $16.937-\mathrm{MHz}$ repetition rate, the per-pulse energy is about 80-pJ. It also has been tested that this TDF laser is capable of long-term operation under mode-locked working mode. Further increase of the pump power will push the laser to operate under higher harmonic modes, for which multi pulses will be generated per round trip.

In summary, an all-fiber GSA mode-locked TDF laser ring oscillator is demonstrated. The laser is pumped by the amplified spontaneous emission source at $1.55-\mu \mathrm{m}$; stable mode-locking operation was achieved with pump power $>130$-mW with low time jittering operation. Soliton pulses with 2.1-ps pulse width were produced centering at 1953.3nm with a $2.2-n m$ FWHM bandwidth. This paper shows that single-layer or few-layer graphene films are effective saturable absorber at $2-\mu \mathrm{m}$. Based on the demonstrated results of this paper, it is possible to further extend functionalities of graphene film into mid-IR photonics.

This work was supported by the National Science Foundation (CMMI-0644681, CMMI-0900564, and CMMI0900419).

${ }^{1}$ K. Tamura, H. Haus, and E. Ippen, "Self-starting additive pulse modelocked erbium fibre ring laser," Electron. Lett. 28, 2226-2227 (1992).

${ }^{2}$ F. ö. Ilday, J. R. Buckley, W. G. Clark, and F. W. Wise, "Self-similar evolution of parabolic pulses in a laser," Phys. Rev. Lett. 92, 213902 (2004).

${ }^{3}$ L. E. Nelson, E. P. Ippen, and H. A. Haus, "Broadly tunable sub-500 fs pulses from an additive-pulse mode-locked thulium-doped fiber ring laser," Appl. Phys. Lett. 67, 19-21 (1995).

${ }^{4}$ Q. Wang, T. Chen, B. Zhang, A. P. Heberle, and K. P. Chen, “All-fiber passively mode-locked thulium-doped fiber ring oscillator operated at solitary and noiselike modes," Opt. Lett. 36, 3750-3752 (2011).

${ }^{5}$ R. C. Sharp, D. E. Spock, N. Pan, and J. Elliot, "190-fs passively modelocked thulium fiber laser with a low threshold," Opt. Lett. 21, 881-883 (1996).

${ }^{6}$ M. A. Solodyankin, E. D. Obraztsova, A. S. Lobach, A. I. Chernov, A. V. Tausenev, V. I. Konov, and E. M. Dianov, "Mode-locked $1.93 \mu \mathrm{m}$ thulium fiber laser with a carbon nanotube absorber," Opt. Lett. 33, 1336-1338 (2008).

${ }^{7}$ K. Kieu and F. W. Wise, "Soliton thulium-doped fiber laser with carbon nanotube saturable absorber," IEEE Photon. Technol. Lett. 21, 128-130 (2009).

${ }^{8}$ Z. Sun, T. Hasan, F. Torrisi, D. Popa, G. Privitera, F. Wang, F. Bonaccorso, D. M. Basko, and A. C. Ferrari, "Graphene mode-locked ultrafast laser," ACS Nano 4, 803-810 (2010).

${ }^{9}$ Q. Bao, H. Zhang, Y. Wang, Z. Ni, Y. Yan, Z. X. Shen, K. P. Loh, and D. Y. Tang, "Atomic-layer graphene as a saturable absorber for ultrafast pulsed lasers," Adv. Funct. Mater. 19, 3077-3083 (2009).

${ }^{10}$ H. Zhang, D. Y. Tang, L. M. Zhao, Q. L. Bao, and K. P. Loh, "Large energy mode locking of an erbium-doped fiber laser with atomic layer graphene," Opt. Express 17, 17630-17635 (2009).

${ }^{11}$ L. M. Zhao, D. Y. Tang, H. Zhang, X. Wu, Q. Bao, and K. P. Loh, "Dissipative soliton operation of an ytterbium-doped fiber laser mode locked with atomic multilayer graphene," Opt. Lett. 35, 3622-3624 (2010).

${ }^{12}$ B. V. Cunning, C. L. Brown, and D. Kielpinski, "Low-loss flake-graphene saturable absorber mirror for laser mode-locking at sub-200-fs pulse duration," Appl. Phys. Lett. 99, 261109 (2011).

${ }^{13}$ J. Liu, Y. G. Wang, Z. S. Qu, L. H. Zheng, L. B. Su, and J. Xu, "Graphene oxide absorber for $2 \mu \mathrm{m}$ passive mode-locking $\mathrm{Tm}: \mathrm{YAlO}_{3}$ laser," Laser Phys. Lett. 9, 15-19 (2012).

${ }^{14}$ G. Q. Xie, J. Ma, P. Lv, W. L. Gao, P. Yuan, L. J. Qian, H. H. Yu, H. J. Zhang, J. Y. Wang, and D. Y. Tang, "Graphene saturable absorber for Q-switching and mode locking at $2 \mu \mathrm{m}$ wavelength," Opt. Mater. Express 2, 878-883 (2012). 
${ }^{15}$ M. Lotya, P. J. King, U. Khan, S. De, and J. N. Coleman, "High-concentration, surfactant-stabilized graphene dispersions," ACS Nano 4, 3155-3162 (2010).

${ }^{16}$ A. Martinez, K. Fuse, B. Xu, and S. Yamashita, "Optical deposition of graphene and carbon nanotubes in a fiber ferrule for passive mode-locked lasing," Opt. Express 18, 23054-23061 (2010).
${ }^{17}$ Q. Ye, C. Xu, X. Liu, W. H. Knox, M. F. Yan, R. S. Windeler, and B. Eggleton, "Dispersion measurement of tapered air-silica microstructure fiber by white-light interferometry," Appl. Opt. 41, 4467-4470 (2002).

${ }^{18}$ S. M. J. Kelly, "Characteristic sideband instability of periodically amplified average soliton," Electron. Lett. 28, 806-807 (1992). 